\title{
SELECTION LARVAIRE ET FREQUENCE GENIQUE CHEZ DROSOPHILA MELANOGASTER
}

\author{
DOMINIQUE ANXOLABEHERE \\ Laboratoire de Biologie Animale 3, Faculté des Sciences de Paris, 12 rue Cuvier-75-Paris, 5ème
}

Received 29.i.70

\section{INTRODUCTION}

LE rôle de la sélection larvaire dans la variation des coefficients sélectifs chez Drosophila melanogaster a été décrit dans le cas de la concurrence entre le gène white et son allèlomorphe sauvage (Petit, 1966) et dans celui de la concurrence entre les estérases du locus 6 (Kojima et Yarbrough, 1967). Dans les cas étudiés, la valeur sélective des larves de chaque génotype est fonction de leur fréquence dans la population larvaire: des types désavantagés quand ils sont abondants voient ce désavantage diminuer quand ils deviennent rares-c'est le cas de white-ou même se transformer en avantage-c'est le cas de l'allélomorphe $\mathrm{F}$ du locus 6--

Il importait donc le vérifier le caractère général du phénomène en examinant différents mutants et de préciser, en vue d'une recherche ultérieure de son mécanisme, quelques uns des facteurs susceptibles de l'influencer.

\section{Materiel et techniques}

\section{(a) Materiél biologique}

Les souches de Drosophila melanogaster utilisées sont presque toutes homogènes: les souches sepia, ebony, white $G$, wiso + et + iso $w$, sont conservée au laboratoire depuis plusieurs années; chacune de ces souches est donc relativement homogène et, de plus, bien adaptée aux conditions d'élevage au laboratoire. Seule, Tautavel, récemment capturée dans la nature, et génétiquement hétérogène.

La concurrence est donc réalisée, dans tous les cas, sauf un sur lequel nous reviendrons, entre des souches différant non seulement par un gène marqueur, mais, par l'ensemble du génôme. L'exception est constituée par les souches $w$ iso + et + iso $w$, en principle isogéniques et ne différant que par la mutation white.

\section{(b) Techniques expérimentales}

Deux techniques ont été employées et leurs résultats comparés.

$\left(1^{\circ}\right)$ Technique d'étalement. Cent œufs des deux génotypes mis en compétition sont placés sur une petite quantité de milieu de Peari $\left(2 \mathrm{~cm} .{ }^{3}\right)$ ensemencé de $0.3 \mathrm{~cm} .^{3}$ d'une solution de levure à 12.5 per cent. Plusieurs séries d'expériences correspondant à des pourcentages d'œufs allant de 10 à 90 per cent. sont réalisées; chaque série est répétée de 5 à 10 fois. Le développement se fait à $25^{\circ} \pm 1^{\circ} \mathrm{C}$. Une hygrométrie relative assez élevée est maintenue par addition tous les 3 jours de quelques gouttes d'eau. Les imagos éclos sont dénombrés chaque jour. 
$\left(2^{\circ}\right)$ Technique de l'ensemencement massif. Vingt femelles fécondées, âgées de 4 jours, sont mises à pondre 65 heures à $25^{\circ} \mathrm{C}$. sur $20 \mathrm{~cm} .^{3}$ de milieu de Pearl ensemencé de $2 \mathrm{~cm} .^{3}$ d'une solution de levure fraiche à 25 per cent. Dans chaque série, la fréquence de femelles mutantes est de $0 \cdot 10 ; 0 \cdot 45 ; 0 \cdot 75$. Chaque jour, les imagos éclos sont dénombrés. Gette technique dont l'avantage est d'obtenir des populations larvaires nombreuses présente néanmoins des inconvénients certains: elle amène à évaluer non plus les seules différences de résistance larvaire, mais aussi les différences de fécondité des femelles. En outre, les différences d'âge entre les larves risquent d'être considérables puisque la ponte s'échelonne sur 65 heures. Elle a néanmoins été utilisée pour une série d'expériences dans lesquelles les résultats obtenus se sont révélés comparables à ceux des témoins faits par la technique d'étalement.

\section{(c) Evaluation des coefficients et techniques statistiques}

Des coefficients ont été évalués pour chacune de ces techniques. Pour analyser les résultats de la technique d'étalement, un coefficient de sélection larvaire est calculé pour chacune des formes. Il est égal au rapport du nombre d'imagos éclos au nombre d'œufs déposés; bien entendu, un coefficient correctif tenant compte du nombre total d'œufs déposés et du nombre total d'imagos éclos, est introduit dans le calcul : $a$ étant le nombre d'œufs de la forme 1, $b$ le nombre d'œufs de la forme 2, $A$ le nombre d'imagos 1 éclos, $B$ le nombre d'imagos 2 éclos, le coefficient de sélection larvaire de la forme 1 sera:

$$
S_{1}=\frac{A}{a} \times \frac{a+b}{A+B}, \text { où } \frac{a+b}{A+B}
$$

est le coefficient correctif. Gette formule peut s'écrire

$$
S_{1}=\frac{\frac{A}{A+B}}{\frac{a}{a+b}}=\frac{P}{p} .
$$

L'erreur standard sur ce coefficient correspond à l'erreur sur le pourcentage d'imagos éclos et non sur un rapport de pourcentages puisque $\frac{a}{a+b}$ est fixé par l'expérimentateur. Elle est égale à: $\frac{A . B}{(A+B)^{3}}$. La valeur sélective relative de la forme 1 par rapport à la forme 2 est donnée par le rapport des coefficients de sélection larvaire:

$$
S_{1 / 2}=\frac{S_{1}}{S_{2}}=\frac{A / a}{B / b}
$$

L'erreur approchée sur ce coefficient, calculée par L'Heritier (Petit, 1958) est égale à:

$$
\pm S_{1 / 2} \frac{(A+B)}{A \cdot B}
$$


Des coefficients peuvent être calculés pour la technique de l'ensemencement massif, mais ils ont une valeur biologique différente. Le rapport $\mathrm{du}$ nombre d'imagos éclos sur le nombre de femelles du même type mises à pondre représente la productivité d'une femelle du génotype considéré, puisqu'il inclut non seulement la résistance larvaire mais aussi la fécondité.

La valeur sélective d'un génotype par rapport à l'autre sera donnée par le rapport des productivités des deux types de femelles. Si $a$ et $b$ sont les nombres de femelles de chaque forme mises à pondre ensemble, $A$ et $B$ les nombres d'imagos correspondants éclos, les productivité des femelles sont $A / a$ et $B / b$; la valeur sélective relative de la forme 1 est donc: $\frac{A / a}{B / b}$.

\section{Resultats}

\section{(a) Influence de la fréquence génique sur les valeurs sélectives}

Toutes ces expériences ont été réalisées par la technique de l'étalement.

(1) Compétition larvaire entre les souches sepia et + iso $w$. Les résultats présentés dans le tableau 1 et les figs. $l a$ et $1 b$, montrent que le coefficient de sélection larvaire de la forme sauvage est d'autant plus élevé que les larves

Tableau 1

Concurrence entre les souches se $e t+$ iso w.

$(\mathrm{P} / \mathrm{p}$ et $\mathrm{Q} / q$ représentent les valeurs sélectives de sepia et de + iso $\mathrm{w}, \mathrm{S}$, la valeur sélective relative de sepia par rapport à + iso $\mathrm{w}$ ).

\begin{tabular}{|c|c|c|c|c|c|}
\hline $\begin{array}{l}\% \text { D'cuufs se } \\
\text { déposés }\end{array}$ & $\begin{array}{l}\text { Nombre d'im- } \\
\text { agos se éclos }\end{array}$ & $\begin{array}{l}\text { Nombre d'im- } \\
\text { agos+éclos }\end{array}$ & $s e: \frac{P}{p} \pm e$ & $+: \frac{Q}{q} \pm e$ & $S_{\mathrm{r}} \pm e$ \\
\hline 10 & $\begin{array}{l}6 \\
5 \\
6 \\
4 \\
6\end{array}$ & $\begin{array}{l}54 \\
57 \\
61 \\
55 \\
76\end{array}$ & $\begin{array}{l}1 \cdot 00 \pm 0.03 \\
0.80 \pm 0.03 \\
0.89 \pm 0.03 \\
0.67 \pm 0.03 \\
0.73 \pm 0.02\end{array}$ & $\begin{array}{l}1.00 \pm 0.03 \\
1.02 \pm 0.03 \\
1.01 \pm 0.03 \\
1.03 \pm 0.03 \\
1.02 \pm 0.02\end{array}$ & $\begin{array}{l}1 \cdot 00 \pm 0.43 \\
0 \cdot 78 \pm 0.36 \\
0.88 \pm 0.37 \\
0 \cdot 65 \pm 0.33 \\
0.72 \pm 0.30\end{array}$ \\
\hline Moyennes & & & $0.81 \pm 0.01$ & $1.02 \pm 0.01$ & $0 \cdot 81 \pm 0 \cdot 16$ \\
\hline 50 & $\begin{array}{l}22 \\
27 \\
14 \\
23 \\
27\end{array}$ & $\begin{array}{l}35 \\
40 \\
47 \\
38 \\
31\end{array}$ & $\begin{array}{l}0.77 \pm 0.06 \\
0.80 \pm 0.05 \\
0.45 \pm 0.05 \\
0.75 \pm 0.06 \\
0.93 \pm 0.06\end{array}$ & $\begin{array}{l}1 \cdot 22 \pm 0.06 \\
1 \cdot 19 \pm 0.05 \\
1 \cdot 54 \pm 0.05 \\
1 \cdot 24 \pm 0.06 \\
1 \cdot 06 \pm 0.06\end{array}$ & $\begin{array}{l}0.62 \pm 0.17 \\
0.67 \pm 0.16 \\
0.29 \pm 0.09 \\
0.60 \pm 0.15 \\
0.87 \pm 0.22\end{array}$ \\
\hline Moyennes & & & $0.74 \pm 0.02$ & $1 \cdot 25 \pm 0 \cdot 02$ & $0.59 \pm 0.06$ \\
\hline Moyennes & $\begin{array}{l}24 \\
37 \\
38 \\
36 \\
21\end{array}$ & $\begin{array}{r}4 \\
8 \\
8 \\
10 \\
9\end{array}$ & $\begin{array}{l}0.95 \pm 0.06 \\
0.91 \pm 0.05 \\
0.91 \pm 0.05 \\
0.86 \pm 0.06 \\
0.77 \pm 0.08 \\
0.88 \pm 0.02\end{array}$ & $\begin{array}{l}1 \cdot 42 \pm 0.06 \\
1 \cdot 77 \pm 0.05 \\
1 \cdot 73 \pm 0.05 \\
2 \cdot 17 \pm 0.06 \\
3.00 \pm 0.06 \\
2 \cdot 00 \pm 0.02\end{array}$ & $\begin{array}{c}0.66 \pm 0.36 \\
0.51 \pm 0.20 \\
0.52 \pm 0.20 \\
0.40 \pm 0.14 \\
0.25 \pm 0.10 \\
0.44 \pm 0.07\end{array}$ \\
\hline
\end{tabular}

sauvages sont rares dans la population; celui de sepia semble au contraire indépendant de la fréquence génique. La valeur sélective relative de sepia par rapport à sauvage varie en fonction de la fréquence génique; sepia est toujours désavantagé, mais son désavantage est moindre quand il est rare que lorsqui'il est abondant.

(2) Compétition larvaire entre les souches ebony et + iso w. Dans cette expérience la variation du coefficient de sélection est identique à celle constantée pour sepia (tableau 2, figs. $2 a$ et $2 b$ ). Mais alors que dans le cas 


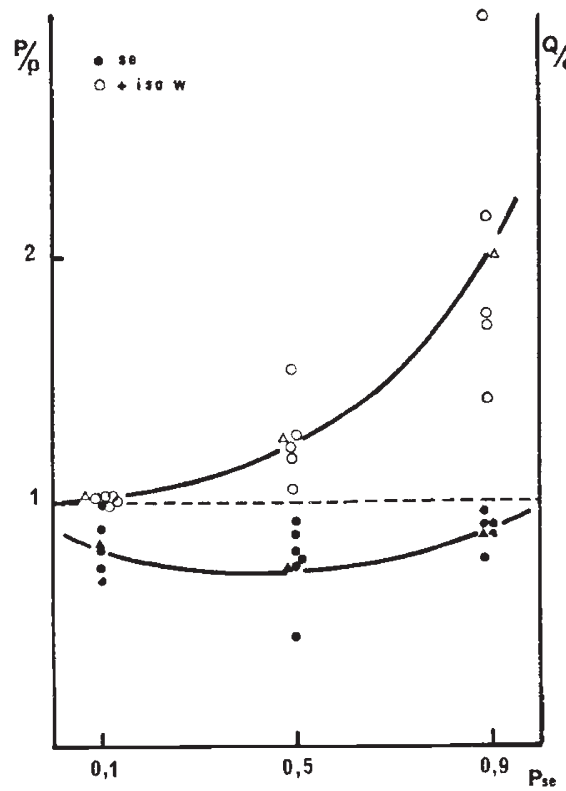

Fig. $1 a$.

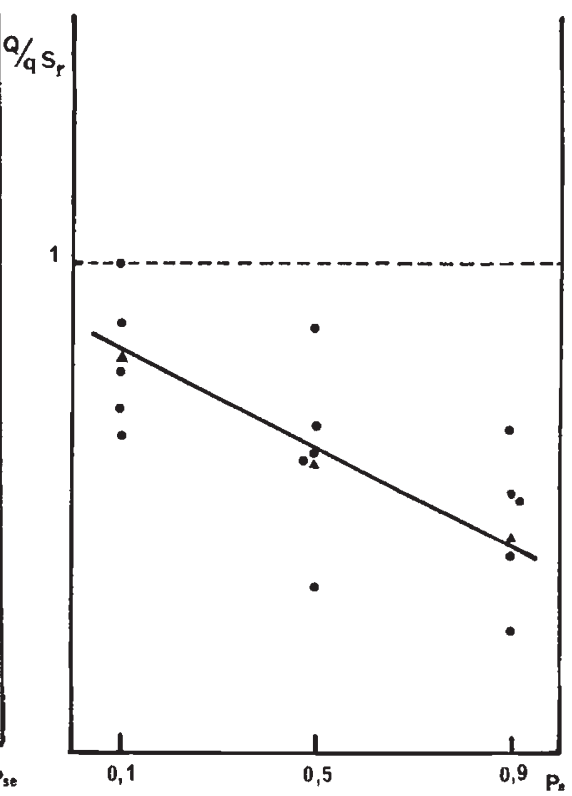

Fig. $1 b$.

Frg. 1.-Concurrence larvaire entre les souches sepia et $+i$ iso $w$.

$1 a-$ Valeurs sélectives absolues $(P / p$ ou $Q / q)$.

$1 b$-Valeurs sélectives relatives $\left(S_{r}\right)$.

(Les moyennes, sont pour chaque fréquence, représentées par un triangle.)

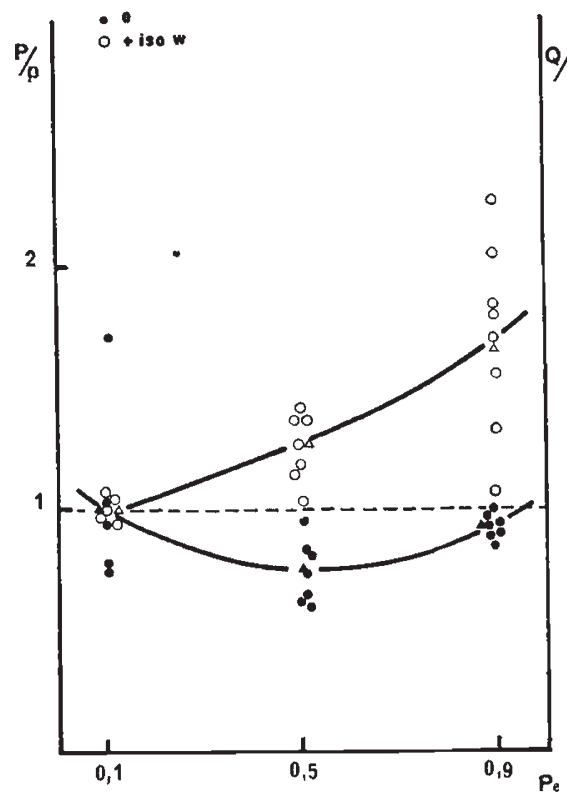

Fig. $2 a$.

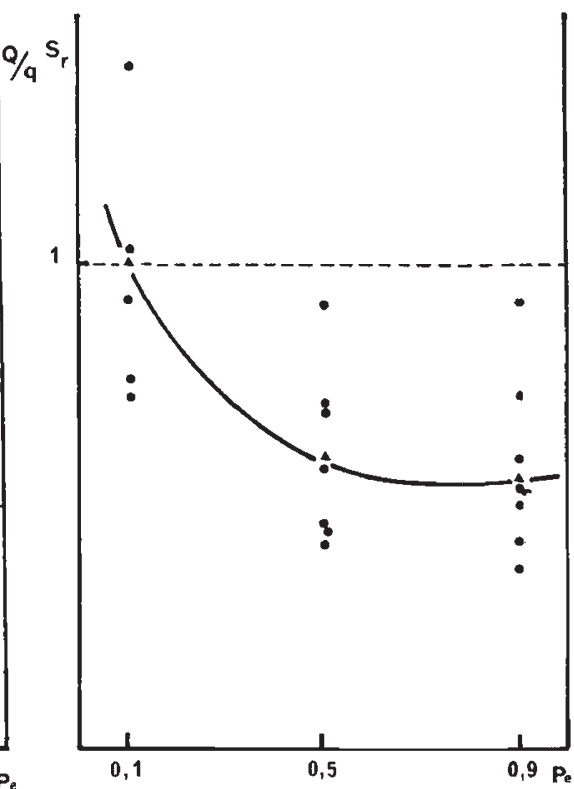

Fig. $2 b$.

Frg. 2.-Concurrence larvaire entre les souches ebony et $+i$ so $w$.

$2 a$-Valeurs sélectives absolues.

$2 b$-Valeurs sélectives relatives. 
de sepia le phénomène ne fait que ralentir l'élimination du gène, il peut ici assurer son maintien: la valeur sélective d'ebony qui augmente lorsque ce gène devient rare, dépasse 1 pour les fréquences inférieures à $0 \cdot 10$. Ceci traduit un advantage d'ebony sur la forme sauvage.

(3) Compétition larvaire entre les souches white et Tautavel. La concurrence larvaire entre les souches white et sauvage a été décrite par Petit (1966). Elle a été reprise avec d'autres souches: les résultats présentés dans le tableau 2 et dans les figs. $3 a$ et $3 b$, diffèrent quelque peu de ceux obtenus avec les souches $w$ iso + et $M 4 C 2$ : la valeur sélective relative de white $G$, inversement proportionnelle à sa fréquence, est supérieure à 1 pour toutes les fréquences

TABleau 2

Influence de la fréquence génique sur les valeurs sélectives

\begin{tabular}{|c|c|c|c|c|c|c|c|}
\hline Lignées 1 et 2 & $\%$ & Répliques & A & B & $P / p \pm e$ & $Q / q \pm e$ & $S_{\mathrm{r}} \pm e$ \\
\hline se et + iso $w$ & $\begin{array}{l}10 \\
50 \\
90\end{array}$ & $\begin{array}{l}5 \\
5 \\
5\end{array}$ & $\begin{array}{r}27 \\
113 \\
156\end{array}$ & $\begin{array}{r}303 \\
191 \\
39\end{array}$ & $\begin{array}{l}0.81 \pm 0.01 \\
0.74 \pm 0.02 \\
0.88 \pm 0.02\end{array}$ & $\begin{array}{l}1 \cdot 02 \pm 0.01 \\
1 \cdot 25 \pm 0.02 \\
2 \cdot 00 \pm 0 \cdot 02\end{array}$ & $\begin{array}{l}0.81 \pm 0.16 \\
0.50 \pm 0.06 \\
0.44 \pm 0.07\end{array}$ \\
\hline e et + iso $w$ & $\begin{array}{l}10 \\
50 \\
90\end{array}$ & $\begin{array}{l}5 \\
7 \\
8\end{array}$ & $\begin{array}{r}31 \\
137 \\
333\end{array}$ & $\begin{array}{r}282 \\
225 \\
66\end{array}$ & $\begin{array}{l}1 \cdot 00 \pm 0.01 \\
0.75 \pm 0.02 \\
0.92 \pm 0.01\end{array}$ & $\begin{array}{l}1 \cdot 00 \pm 0.01 \\
1 \cdot 24 \pm 0.02 \\
1 \cdot 65 \pm 0.01\end{array}$ & $\begin{array}{l}1 \cdot 00 \pm 0.18 \\
0 \cdot 60 \pm 0.06 \\
0.56 \pm 0.07\end{array}$ \\
\hline$v_{G}$ et $+i s o w$ & $\begin{array}{l}10 \\
50 \\
90\end{array}$ & $\begin{array}{l}4 \\
5 \\
2\end{array}$ & $\begin{array}{r}25 \\
103 \\
82\end{array}$ & $\begin{array}{l}84 \\
51 \\
10\end{array}$ & $\begin{array}{l}2 \cdot 29 \pm 0.04 \\
1 \cdot 33 \pm 0.03 \\
0.99 \pm 0.03\end{array}$ & $\begin{array}{l}0.85 \pm 0.04 \\
0 \cdot 66 \pm 0.03 \\
1 \cdot 20 \pm 0.03\end{array}$ & $\begin{array}{l}2 \cdot 67 \pm 0.61 \\
2 \cdot 01 \pm 0.34 \\
0.91 \pm 0.30\end{array}$ \\
\hline$w$ iso + et + iso $w$ & $\begin{array}{l}10 \\
50 \\
90\end{array}$ & $\begin{array}{r}10 \\
9 \\
9\end{array}$ & $\begin{array}{r}5 \\
113 \\
360\end{array}$ & $\begin{array}{r}598 \\
321 \\
78\end{array}$ & $\begin{array}{l}0.08 \pm 0.01 \\
0.52 \pm 0.02 \\
0.91 \pm 0.01\end{array}$ & $\begin{array}{l}1 \cdot 10 \pm 0 \cdot 01 \\
1 \cdot 47 \pm 0 \cdot 02 \\
1 \cdot 78 \pm 0 \cdot 01\end{array}$ & $\begin{array}{l}0.07 \pm 0.03 \\
0 \cdot 35 \pm 0.03 \\
0.51 \pm 0.06\end{array}$ \\
\hline $\begin{array}{l}\text { iso }+ \text { et } \\
\quad+\text { Tautavel }\end{array}$ & $\begin{array}{l}10 \\
50 \\
90\end{array}$ & $\begin{array}{l}5 \\
4 \\
2\end{array}$ & $\begin{array}{r}8 \\
11 \\
29\end{array}$ & $\begin{array}{r}103 \\
90 \\
11\end{array}$ & $\begin{array}{l}0.72 \pm 0.02 \\
0.22 \pm 0.03 \\
0.81 \pm 0.07\end{array}$ & $\begin{array}{l}1 \cdot 03 \pm 0.02 \\
1 \cdot 78 \pm 0.03 \\
2 \cdot 75 \pm 0.07\end{array}$ & $\begin{array}{l}0.70 \pm 0.25 \\
0.12 \pm 0.03 \\
0.29 \pm 0.10\end{array}$ \\
\hline
\end{tabular}

inférieures à 0.90 ; non seulement la courbe à minimum de Petit ne se retrouve pas mais le désavantage de white a disparu. Ce résultat qui prouverait, s'il en était encore beson, l'influence de l'ensemble du génome sur la concurrence larvaire, s'explique probablement par l'histoire des souches utilisées: alors que les souches de Petit et la souche white $G$ sont des souches dans lesquelles une longue vie au laboratoire a permis une sélection en faveur des génotypes les mieux adaptés, la souche sauvage utilisée ici était de capture récente et certainement mieux adaptée aux conditions de vie naturelle qu'à celles du laboratoire.

(4) Compétition larvaire entre deux souches "isogeniques". On pouvait se demander si le phénomène existait entre deux souches théoriquement isogéniques.

Les résultats de la concurrence larvaire entre la souche wiso + et la souche $+i$ so $w$ sont présentés dans le tableau 2 et sur les figs. $4 a$ et $4 b$. Ici aussi l'intensité de la sélection larvaire dépend de la fréquence génique, mais résultat inattendu et contraire aux précédent, le désavantage du gène white s'accentue à mesure que baisse la fréquence des œufs white dans la population. On pouvait se demander si ce résultat était dû à la souche white 


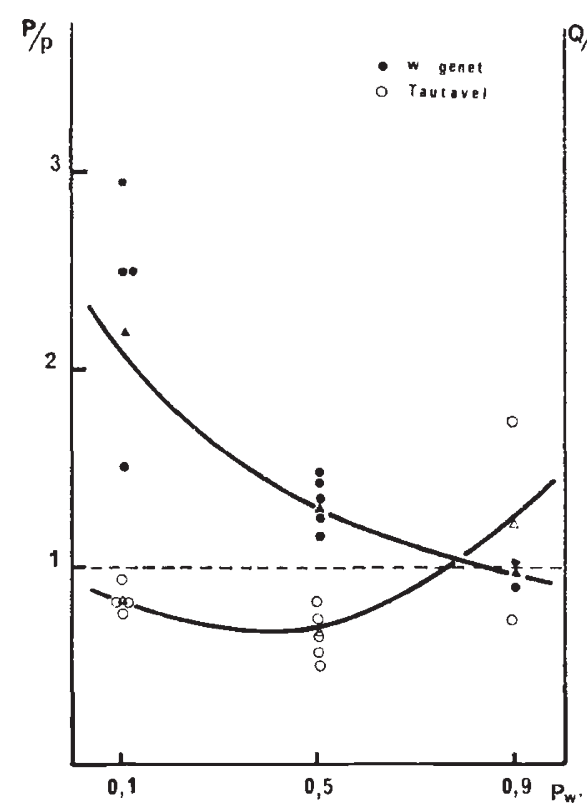

[Frg. 3a.

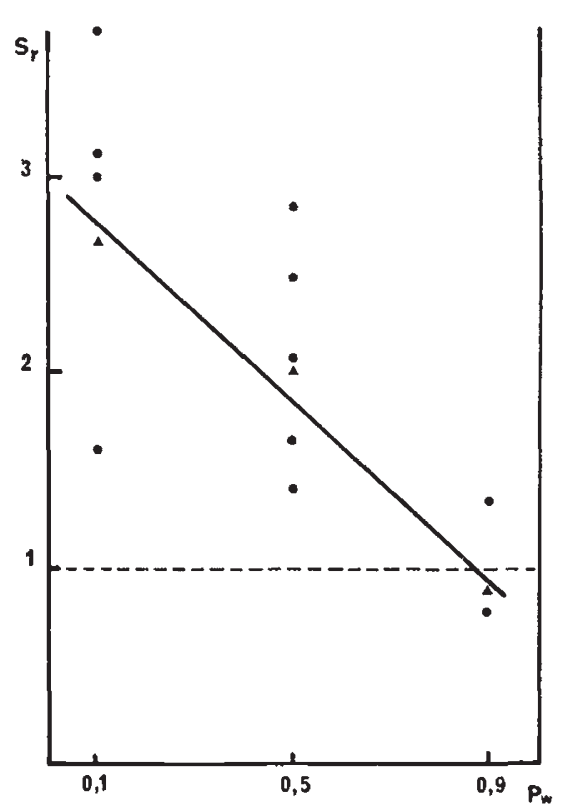

Fig. $3 b$.

Fig. 3.-Concurrence larvaire entre les souches white $G$ et Tautavel.

$3 a$-Valeurs sélectives absolues.

$3 b$-Valeurs sélectives relatives.

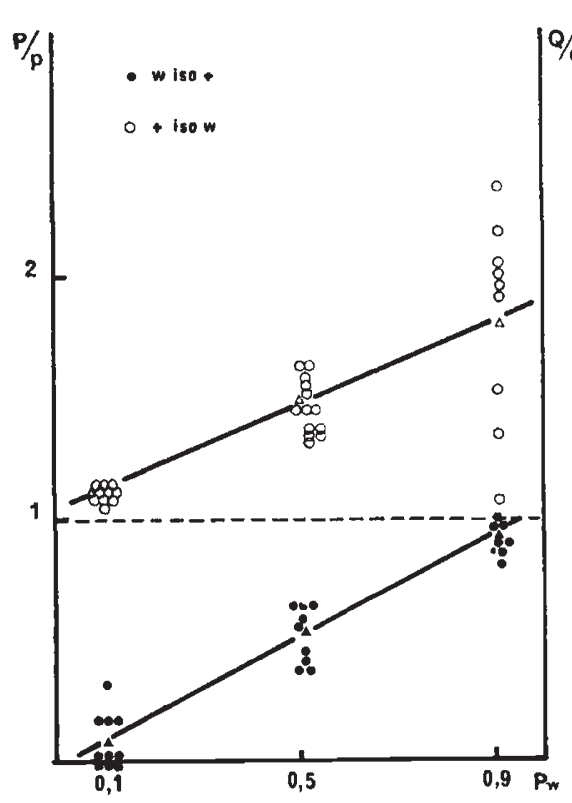

Frg. $4 a$.

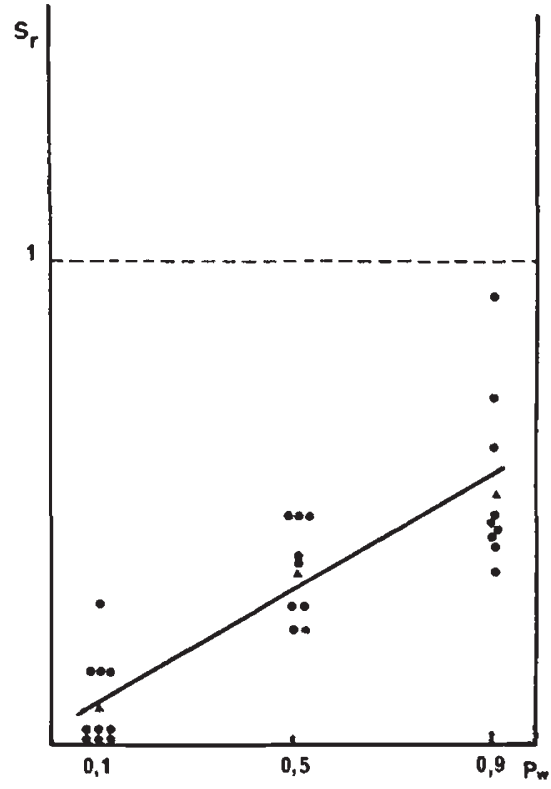

Frg. $4 b$.

Fro. 4.-Concurrence larvaire entre les souches $w$ iso + et + iso $w$. $4 a-$ Valeurs sélectives absolues. $4 b$-Valeurs sélectives relatives. 
utilisée. Elle fut donc mise en concurrence avec une souche sauvage très hétérogène: + Tautavel. Les résultats consignés dans le tableau 2 et la fig. 5 montrent qu'avec cette nouvelle souche l'avantage de la forme rare est retrouvé, avec une courbe à minimum.

\section{(b) Influence de la quantité de nourriture}

L'examen de la compétition larvaire entre deux souches données montre donc une relation certaine entre les valeurs sélectives et la fréquence génique. Cette relation est-elle modifiée en fonction de l'intensité de la sélection, comme les résultats de Sokal et col. relatifs à l'influence de la densité (1963 et 1964) permettent de le penser? La quantité de nourriture mise a la disposition des larves étant un facteur essentiel de l'intensité de la sélection, des expériences ont été réalisées en faisant varier la quantité de levure fraîche.

(1) Compétition entre les souches ebony et + iso w. Dans une première série d'expériences, le milieu de culture est ensemencé avec $0.3 \mathrm{~cm} .{ }^{3} \mathrm{~d}^{\text {'une solution }}$ de levure fraîche à 75 per cent.; dans une seconde série, avec une solution de levure fraîche à 12.5 per cent. Les valeurs sélectives relatives sont présentées sur la fig. 6. L'avantage de la forme rare, retrouvé lorsque la sélection est forte, disparaît, lorsqu'elle devient faible.

(2) Compétition entre les souches $\mathrm{w}$ iso + et + iso $\mathrm{w}$. Les concentrations de levure fraîche utilisées sont 75 per cent. et 25 per cent. Les résultats (fig. 7) sont du même type que précédement, bien que moins nets ce qui est normal puisque la concurrence est moins forte.

Les relations entre les valeurs sélectives et la fréquence génique sont donc considérablement modifiées par l'intensité de la sélection. En particulier le phénomène de la diminution du désavantage de la forme rare disparaît lorsque la quantité de nourriture mise à la disposition de la population larvaire est grande.

\section{(c) Influence de la température}

L'influence de la température sur la variation des coefficients sélectifs à été envisagée uniquement par la technique de l'ensemencement massif. Elle ne permet qu'une étude globale du phénomène, mais exige beaucoup moins de manipulation.

La compétition larvaire entre la souche white $G$ et la souche Tautavel à été étudiée à trois températures différentes: $20^{\circ} \mathrm{C}$. $; 25^{\circ} \mathrm{C}$. et $28^{\circ} \mathrm{C}$. $\mathrm{La}$ fécondité de chacune des deux souches n'ayant pas été évaluée, on ne peut assimiler la fréquence des œufs white à la fréquence des imagos white dans la population des pondeuses. C'est donc la productivité globale de chaque génotype, qui est examinée ici et non la valeur sélective larvaire. De plus les différences de fécondité entraînent une variation de la densité larvaire en fonction des fréquences des deux génotypes.

A $20^{\circ}$ et $25^{\circ}$, la valeur sélective relative de white est plus élevée quand cette forme est rare que lorsqu'elle est abondante, mais cet avantage de la forme rare disparait à $28^{\circ}$ (tableau 3). Notons que la compétition larvaire entre les deux même souches, étudiée à $25^{\circ}$, avec la technique de l'étalement donne des résultats comparables (cf. tableau 2 et fig. $3 b$ ). Ce résultat s'explique probablement par le fait que la concurrence pour l'aliment joue comme facteur limitant. 


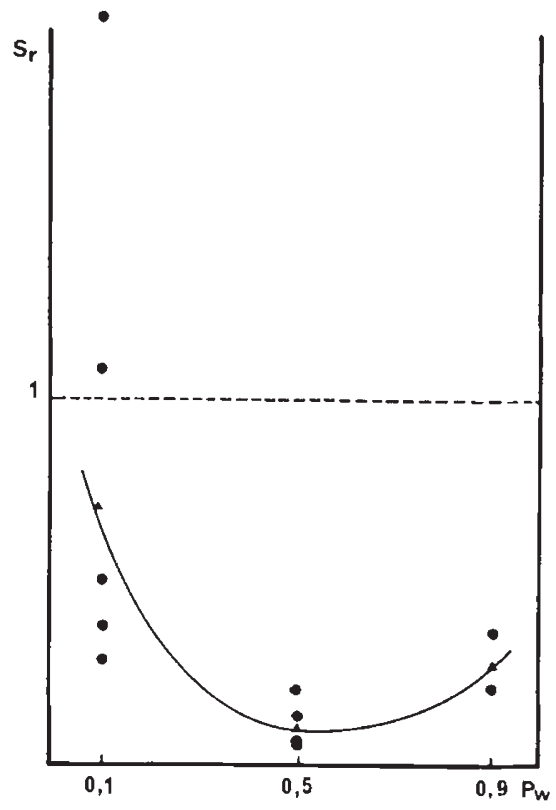

Fig. 5.-Concurrence larvaire entre les souches $w$ iso + et Tautavel.

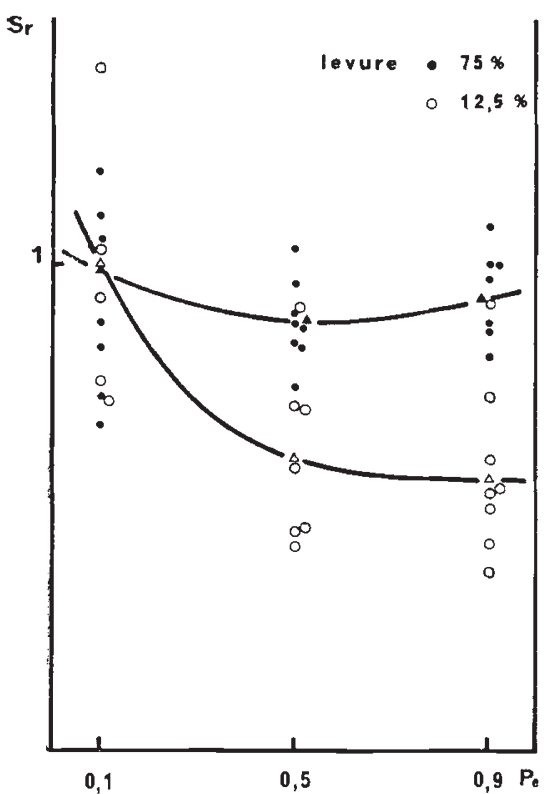

Fig. 6.-Influence de la quantité de nourriture.-Concurrence entre les souches ebony et + iso w.

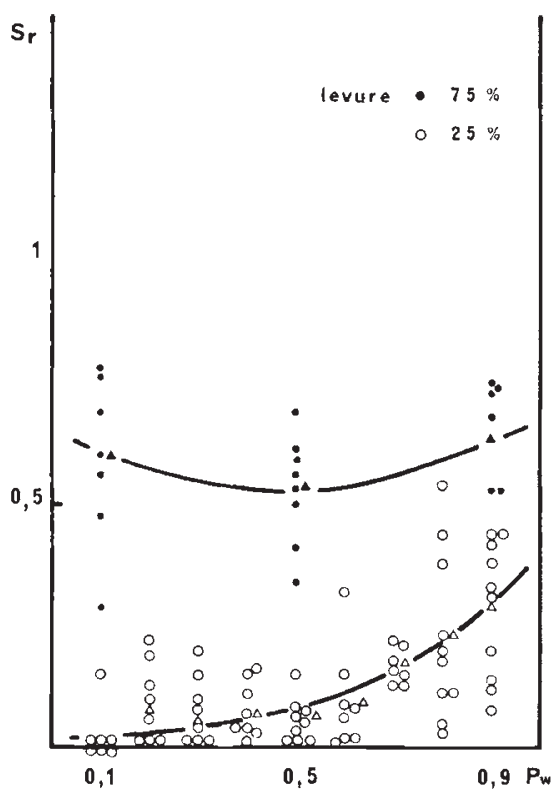

Fro. 7.-Influence de la quantité de nourriture.-Concurrence entre les souches $w$ iso + et + iso $w$. 
Bien que limités, ces résultats indiquent que la température influe sur la relation qui lie les valeurs sélectives à la fréquence génique. Cette action était

Tableau 3

Influence de la température sur les valeurs sélectives

$\begin{array}{cccc}\begin{array}{c}\text { Fréquence de } w \\ \text { chez les mères }\end{array} & S_{r} \text { de } w_{G} \text { à } 20^{\circ} & S_{r} \text { de } w_{G} \text { à } 25^{\circ} & S_{r} \text { de } w_{G} \text { à } 28^{\circ} \\ 0.10 & 1.86 \pm 0.36 & 2 \cdot 87 \pm 0.33 & 0.94 \pm 0.19 \\ 0.45 & 0.57 \pm 0.08 & 0.76 \pm 0.07 & 0.86 \pm 0.09 \\ 0.75 & 0.28 \pm 0.03 & 0.93 \pm 0.09 & 0.58 \pm 0.06\end{array}$

prévisible puisque la température modifie la vitesse de développement larvaire, et que la température optimale d'élevage varie d'une souche à l'autre.

\section{Discussion et conclusion}

Malgré les difficultiés recontrées pour obtenir une bonne répétabilité des expériences, les résultats relatifs au coefficient de sélection larvaire de chaque forme sont relativement satisfaisants. Et si une amélioration est susceptible d'être obtenue par l'utilisation de milieux synthétiques, certaines conclusions peuvent néanmoins être tirées des résultats précédents:

- une variation de l'intensité de la sélection larvaire en fonction de la fréquence des génotypes en présence à été trouvée dans tous les cas examinés,

- cette variation avantage généralement le type rare; une seule exception a été relevée dans la série étudiée.

Cet avantage du type rare, déjà mis enévidence chez Drosophila melanogaster lors de l'étude de la concurrence entre souches marquées (Petit, 1966) et entre deux allèles responsables de la fabrication d'estérases (Kojima et Yarbrough, 1967) a donc été retrouvé dans la presque totalité des souches ou il a été recherché. Déjà, un phénomène comparable avait été mis en évidence par Sokal chez Tribolium castaneum (Sokal et Huber, 1963; Sokal et Karten, 1964).

Bien que le mécanisme du phénomène soit encore inconnu, certaines hypothèses peuvent être envisagées: si, à coté d'un certain nombre de substances nécessaires aux deux formes, existent des substances plus spécialement utiles à chacune d'elles, la concurrence sera d'autant plus vive que le milieu est en quantité plus limitée et la forme considérée plus abondante; mais cette limitation par le milieu disparaît quand le milieu s'enrichit, comme le prouve, l'influence de la quantité de levure.

Il importe donc maintenant de rechercher la nature de ces substances différentes, dont l'éxistence n'apparaît actuellement que comme une nécessité logique. Il faut aussi examiner les interactions entre les formes en concurrence, la composition du milieu variant en fonction de la fréquence des formes qui s'y développent et l'éxistence d'interactions entre génotypes par l'intermédiaire du milieu étant connue (Polivanov, 1964; Dawood et Strickberger, 1964; Weisbrot, 1966).

Quoiqu'il en soit, l'avantage de la larve rare est certainement un cas particulier de l'avantage du type rare dont l'importance pour le maintien du polymorphisme apparaît chaque jour plus clairement. 


\section{RÉSUMÉ}

1. La compétition larvaire entre des souches mutantes $(e$, se, et $w$ ) et sauvage de Drosophila melanogaster a été étudiée pour différentes fréquences géniques; dans tous les cas étudiés on trouve une variation de l'intensité de sélection larvaire en fonction de la fréquence des génotypes en présence, avec le plus souvent, avantage du type rare.

2. La relation entre le succès d'une forme et sa fréquence dans la population larvaire est influencée par la quantité de nourriture disponible et la température de développement.

\section{SUMMARY}

1. Larval competition has been studied between different strains marked by the mutations white, sepia or ebony and several wild strains.

2. When the strains are reared on a limited quantity of nutrient medium, the chances of a larva developing into an imago vary with the strains in competition and are frequency-dependent.

3. The rare type is at an advantage in most cases.

4. The frequency-dependency is influenced by the amount of food and by the temperature during development.

5. Polymorphism could be maintained by this frequency-dependent selection.

\section{Bibliographie}

DAWOOD, M., AND STRICBERGER, M. 1964. The effect of larval interaction on viability in Drosophilia melanogaster. Genetics, 50, 999-1007.

KOJIMA, K., AND YARBROUGH, K. 1967. Frequency dependent selection at the esterase 6-locus in Drosphila melanogaster. Proc. Natl. Acad. Sci. U.S.A., 57, 645-648.

PETIT, c. 1966. La concurrence larvaire et le maintien du polymorphisme. C.R. Acad. Sc., 223, 1263-1265.

PETIT. C. 1958. Le déterminisme génetique et psycho-physiologique de la compétition sexuelle chez Drosophila melanogaster. Bull. Biol., 92, 248-329.

Polivonov, s. 1964. Selection in experimental populations of Drosophila melanogaster with different genetic background. Genetics, 50, 81-100.

SOKAL, R., AND HUBER, I. 1963. Competition among genotypes in Tribolium castaneum at varying densities and gene frequencies (the sooty locus). The American Naturalist, Vol. XGVII, No. 894, 169-184.

SOKAL, R., AND KARTEN, I. 1964. Competition among genotypes in Tribolium castaneum at varying densities and gene frequencies (the black locus). Genetics, 49, 195-211.

WEISBROT, D. R. 1966. Genotypic interactions among competing strains and species of Drosophila melanogaster. Genetics, 53, 427-435. 